\title{
Design of Electronic Transformer Used in a Proposed Circuit Topology for PET
}

\author{
Huina Yang, Xiaodong Wang, Gang Wang \\ School of Electric and Electronic Engineering \\ North China Electric Power University \\ Beijing, China \\ yanghn@ncepu.edu.cn \\ wangxiaodong3182@sina.com \\ wanggang830826@163.com
}

\begin{abstract}
Power Electronic Transformer (PET) is a new type of energy conversion device in power system. The electronic transformers used in PET play an important role in performance of the overall power electronic system, including efficiency, size, and cost. The transformers are high power medium frequency transformer according to present technology. Based on a proposed circuit topology of PET, the design method of the electronic transformer is presented. A $30 \mathrm{KW}$ medium frequency transformer is designed. The selection of core material was based upon the optimization among the specifications: transformer size, efficiency, and producing cost. Thermal analysis is made by ANSYS. The temperature rise is checked by calculating the distribution of temperature field. The design of electric insulation of the electronic transformer is proved to be good by simulation.
\end{abstract}

Keywords-Circuit topology; Power Electronic Transformer; Transformer design; Simulation

\section{INTRODUCTION}

The drawbacks of conventional power transformers, such as heavy weight, large size, sensitivity to harmonics, are arising many problems with the development of power system. Power Electronic Transformer (PET) is a new type of energy conversion device that can overcome the shortcomings of conventional power transformers. In recent years the technology of power semiconductor device made a great progress, which caused the low cost of switching tube and improved the withstanding voltage level. At the same time, because the PWM algorithms became more and more mature and the higher-speed control processors appeared, PET became a hot subject of research.

Different circuit topologies for PET were researched ${ }^{[1]-[4]}$. The classification of voltage in power system is high to $10 \mathrm{KV}$ in general. Whereas the voltage class of IGBT is about $1 \mathrm{KV}$. So series multiple connection should be adopted. The circuit topologies in [1]-[4] were all based on the idea.

In PET, electronic transformers, which are the magnetic components, are required. According to present technology, these transformers work at medium frequency in the power electric system. The performance of the electronic transformers will influence volume of PET, transportation efficiency, quality of output waveform, and safety of switching tube. The heating problem of the electronic transformers is very serious. So the check of thermal analysis is important. Temperature rise can affect the selecting rationality of transformer core material and insulation material.

Most of current design methods for electronic transformer are only suitable for designing low power transformer which temperature rises are no more than $50^{\circ} \mathrm{C}^{[5]-[7]}$. But the temperature rise of the electronic transformer used in PET usually more than $100^{\circ} \mathrm{C}$. In [5] and [6], transformer core and winding are treated as equivalent thermal resistances respectively, and temperature rise is calculated according to the relationship between transformer thermal resistance and temperature rise. In [7], temperature rise is estimated by using the curve of area product - surface area - temperature rise or an approximate formula. But these methods referred in [5]-[7] hold well for temperature rise only in range from $20^{\circ} \mathrm{C}$ to $50^{\circ} \mathrm{C}$. When temperature rise is more than $50^{\circ} \mathrm{C}$, biggish error will occur by these traditional methods to estimate temperature rise.

In this paper, a circuit topology for PET is proposed, which can be used for power transformation in medium and high voltage grid. Based on the proposed circuit topology, the design method of the electronic transformer used in PET is established and the design of a $30 \mathrm{KW}$ transformer, operating at $5000 \mathrm{~Hz}$, is shown in the paper. The simulating result of temperature field of the designed transformer shows that the materials of core and insulation can meet the demand of temperature rise. The simulating results about dielectric strength test of the designed transformer are presented. Based on the distributions of electric field, the electric insulation is checked.

\section{CIRCUIT TOPOLOGY}

The configuration of the circuit topology is shown as Fig. 1. It is for a step-down PET. The features of the proposed circuit topology are as follows:

(1) The power transformation can be performed in medium and high voltage system.

(2) The structure is extremely modular by connecting the same power module.

(3) Reversible flow of power can be realized. 010.

This work was supported by State Grid Corporation of China DL71-09- 
As shown in Fig. 1, $u_{i n}$ is the voltage of power supply system. $u_{\text {out }}$ is the voltage of electric equipment. The waveforms of them are sinusoidal form. The IGBT is switching tube. $u_{1}$ and $u_{2}$ are the input and output voltages of the electronic transformer respectively. The waveforms of them are alternating square waves and have the same frequency. With the increase of the working frequency, the size of the electronic transformer will be reduced. But the loss will increase at the same time. The size and loss of PET are decided mainly by the electronic transformer. So there should be a compromise in choosing the frequency of the electronic transformer. And considering the working frequency of IGBT, the frequency was determined to be $3 \mathrm{KHz}-5 \mathrm{KHz}$.

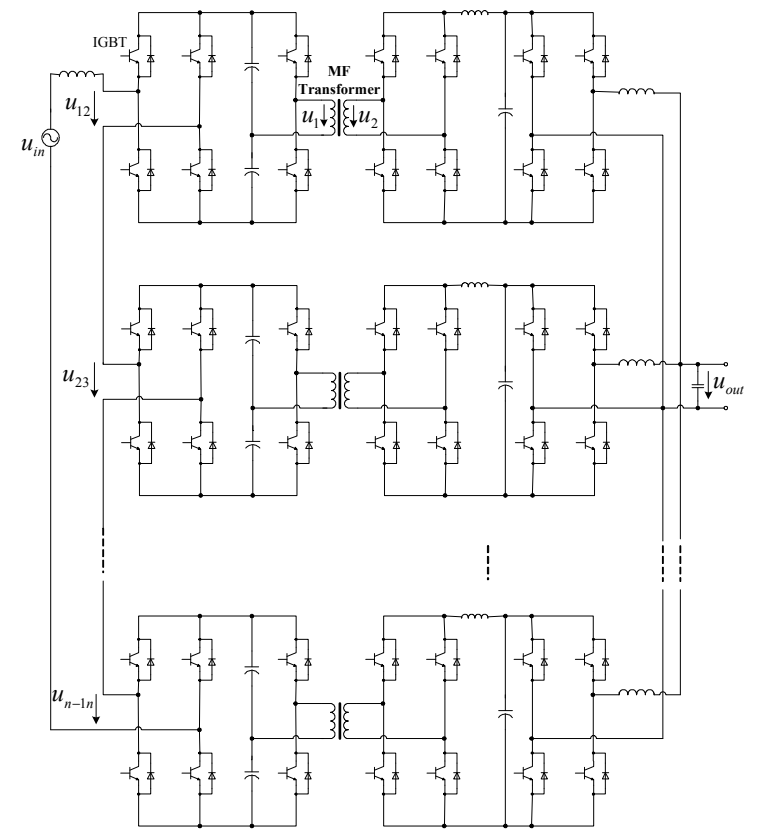

Figure 1. Configuration of circuit topology

\section{DESIGN OF THE MEDIUM FREQUENCY TRANSFORMER}

The electronic transformer is the most important part of the PET for it determines the performance and power conversion efficiency of the PET, as well as size and cost of the whole system. So the optimal design of the electronic transformer is a critical step. Due to the interdependence and interaction of parameters, some tradeoffs have to be made in order to get allowable transformer size, minimum efficiency which can be tolerated, and low producing cost.

\section{A. Selection of Core Material}

The characteristic demand of the core material of the electronic transformer is higher flux density, higher Curie temperature, lower loss, and lower cost. The choice of core material is based on achieving the best characteristic on important design parameters and on acceptable compromises on other parameters ${ }^{[8]}$.

Soft ferrite is the most common core material of low power medium-high frequency transformer. But it is not suitable for high power transformer. Due to its very low flux density in magnetic material, the size of the core would be too bulky. On the other hand, the Curie temperature of Soft ferrite is very low, which results in low working temperature of magnetic core. By property contrast, amorphous alloys and super microcrystalline are thought to be the best choices of core material. But there is not the uniform national standard of magnetic core that is made of amorphous alloys or super microcrystalline at present, and the producing costs of these materials are high. Silicon steel has been greatly improved in past decade, Cold-rolled, grainoriented, thin type silicon steel has the high Curie temperature and flux density close to amorphous alloys and super microcrystalline ${ }^{[8]}$. And the producing cost of silicon steel is the lower than amorphous alloys and super microcrystalline. The shortcoming is that the loss of silicon steel core is higher comparatively. But it can be accepted. Considering all the factors, silicon steel is chosen as the core material of the electronic transformer.

\section{B. Determination of Core Size}

In the design of the electronic transformer, the required parameters are as follows:

Output power: $P_{o}$

Frequency: $f$

Input voltage: $U_{1}$

Minimum input voltage: $U_{1 \min }$

Output voltage: $U_{2}$

On time: $T_{o n}$

The apparent power, $P_{t}$, can be calculated by following equation:

$$
P_{t}=P_{o}\left(1+\frac{1}{\eta}\right)
$$

$\eta$ : efficiency, 95\%-99\%

$P_{t}$ : apparent power ( W )

The area product can be calculated by [8]

$$
A_{P}=\frac{P_{t} \times 10^{2}}{4 K_{m} f B_{m} j}
$$

$A_{p}$ : area product $\left(\mathrm{mm}^{4}\right)$

$B_{m}$ : flux density ( $\left.\mathrm{mT}\right)$

$j$ : current density $\left(\mathrm{A} / \mathrm{mm}^{2}\right), j=3-5 \mathrm{~A} / \mathrm{mm}^{2}$ in initial selection

$K_{m}$ : window utilization factor, $K_{m}=0.2-0.3$

C. Calculation of the winding number

The primary turns can be calculated by

$$
N_{1}=\frac{U_{1} T_{o n}}{2 B_{m} A_{e}} \times 10^{-2}
$$

Where

$$
\begin{aligned}
& N_{1}: \text { primary turns } \\
& T_{o n}: \text { on time }(\mu \mathrm{s}) \\
& A_{e}: \text { core cross-section area }\left(\mathrm{cm}^{2}\right)
\end{aligned}
$$


The secondary turns can be calculated by

$$
N_{2}=\frac{U_{2}}{U_{1}} N_{1}
$$

\section{Selection of Winding Model}

The primary current can be calculated by

$$
I_{1}=\frac{P_{o}}{U_{1 \min } \eta}
$$

The secondary current can be calculated by

$$
I_{2}=\frac{N_{1}}{N_{2}} I_{1}
$$

\section{E. Check of Temperature Rise}

When silicon steel is adopted as the transformer core material in the design, the core loss can be comparatively high. Higher core loss will bring higher temperature rise.

The total loss of the electronic transformer, $P_{\Sigma}$, includes core loss and copper loss. The core loss can be calculated by the following relation:

$$
P_{F e} \propto f^{k_{1}} B_{m}^{k_{2}}
$$

$P_{F e}:$ core loss

$k_{1}, k_{2}:$ constant

The copper loss can be calculated by

$$
P_{C u}=I_{1}^{2} R_{a c 1}+I_{2}^{2} R_{a c 2}
$$

$R_{a c 1}:$ ac resistance of primary winding $(\Omega)$

$R_{a c 2}:$ ac resistance of secondary winding $(\Omega)$

Total loss is as follows:

$$
P_{\Sigma}=P_{F e}+P_{C u}
$$

\section{F. Check of electric insulation}

Withstand voltage test should be made after the electronic transformer is built. Electric insulation should be checked when testing voltage is applied on primary winding and transformer core, secondary winding and transformer core, primary winding and secondary winding respectively. It is significant to analyze the dielectric strength of the designed transformer by simulation before the transformer is built.

\section{G. Efficiency calculation of the designed transformer}

The efficiency of the electronic transformer is determined by the following equation:

$$
\eta=\frac{P_{o}}{P_{o}+P_{F e}+P_{C u}}
$$

\section{DESIGN RESUlT FOR A 30KW MEDIUM FREQUENCY TRANSFORMER}

In our work, a $30 \mathrm{KW}$ medium frequency electronic transformer was designed for the PET. The specification of the transformer is shown as follows:

Operation mode: full bridge

Output power: $P_{o}=30 \mathrm{KW}$

Frequency: $f=5000 \mathrm{~Hz}$

Input voltage: $U_{1}=1200 \mathrm{~V}$

Minimum input voltage: $U_{1 \min }=1000 \mathrm{~V}$

Output voltage: $U_{2}=350 \mathrm{~V}$

On time: $T_{o n}=95 \mu \mathrm{s}$

The grain-oriented silicon steel strip was adopted as the core material. The material grade and type of the transformer core were determined according to national standard of the People's Republic of China. The core material grade was 3Q3000 and the core type was EI.

According to equation (3) and equation (4), $N_{1}=52$. $N_{2}=15$. In order to reduce the leakage inductance, the primary winding was spilt on either side of the secondary winding. The primary winding and the secondary winding were all adopted double glass-fiber covered rectangular copper wire. Based on the principle of winding design, single conductor was adopted for the primary winding and two paralleled conductors for secondary winding. The insulation classes of primary and secondary winding were all selected as 2 nd grade.

The efficiency of the designed transformer was $98 \%$ by calculation.

Polytetrafluoroethylene(PTFE) film was used as the layer insulation between the primary and secondary winding. For the dielectric coefficient of PTFE is comparatively low, using PTFE film as the layer insulation between the primary and secondary winding can make distributed capacitance of the designed transformer low.

\section{Simulation Result}

\section{A. Check of Temperature Rise}

Two-dimensional model was adopted. The analysis crosssection is shown in Fig.2. The calculating result is shown in Fig.3. Forced air cooling must be adopted. It can be found that the highest temperature point of the designed transformer locates in the center of the transformer core. The working temperature of the silicon steel core should be under $300^{\circ} \mathrm{C}$. The insulation materials, including PTFE film, impregnating varnish, and fiberglass of winding, were all selected those which working temperatures were over $200^{\circ} \mathrm{C}$ in this design.

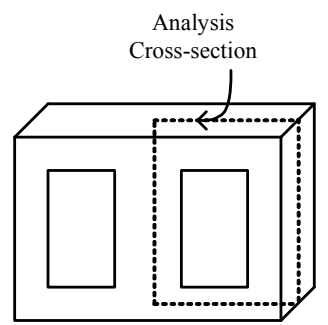

Figure 2. Analysis cross-section 


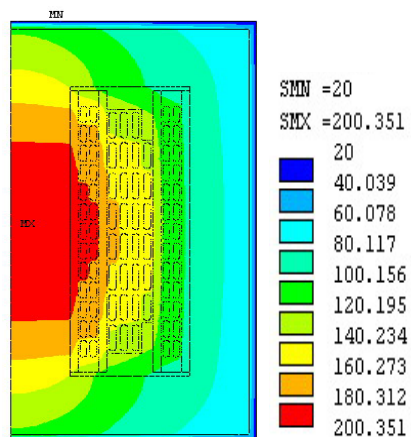

Figure 3. Calculating result of temperature field

\section{B. Check of Electric Insulation}

According to national standard of the People's Republic of China, the testing voltage was $3400 \mathrm{~V}$. The simulating results are shown respectively from Fig.4 to Fig.6. It can be found that the maximal value of electric-field intensity locates in the range between $2.2 \mathrm{KV} / \mathrm{mm}-2.3 \mathrm{KV} / \mathrm{mm}$. The disruptive field intensity of PTFE film ranges in $30 \mathrm{KV} / \mathrm{mm}-60 \mathrm{KV} / \mathrm{mm}$. The disruptive field intensity of impregnating varnish is over $60 \mathrm{KV} / \mathrm{mm}$ in normal state and is over $30 \mathrm{KV} / \mathrm{mm}$ in soaking state. The lowest disruptive field intensity of the glass-fiber was $4 \mathrm{KV} / \mathrm{mm}$. The design of electric insulation is proper.

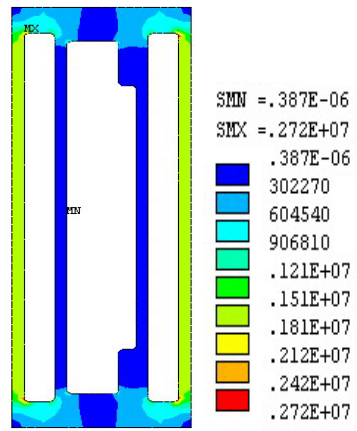

Figure 4. Electric-field distribution when testing voltage is applied on primary winding and core

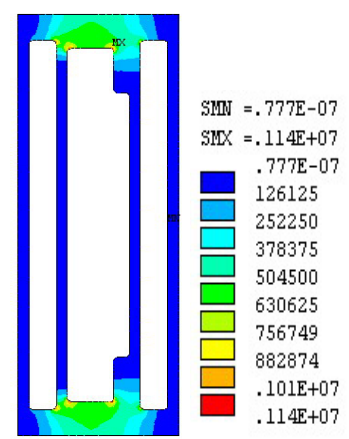

Figure 5. Electric-field distribution when testing voltage is applied on secondary winding and core

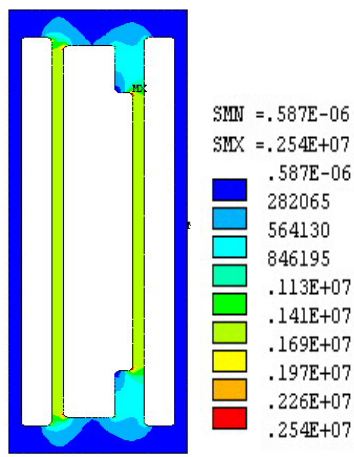

Fig. 6. Electric-field distribution when testing voltage is applied on primary winding and secondary winding

\section{CONCLUSION}

Based on the proposed circuit topology for PET, a design method of high power medium frequency transformer was presented and a $30 \mathrm{KW}$ and $5000 \mathrm{~Hz}$ transformer has been designed according this method. Tradeoffs have been made in the designing process. The checks of temperature rise and electric insulation have been made by ANSYS. The results indicate that the types of all insulation materials can be determined by simulation results of electric-field, and the temperature index of insulation materials and the coefficient of the thermal conductivity of impregnating varnish can be determined by simulation result of temperature field. The design method is suitable for designing the high power electronic transformer used in PET.

\section{REFERENCES}

[1] Edward R. Roman, Scott D Sudhoff, Steven F. Glover and Dudley L. Galloway, "A Power Electronic-Based Distribution Transformer," IEEE Trans. Power Delivery, Vol.17, No. 2, pp.537-543, April 2002.

[2] H. Iman-Eini and S. Farhangi, "Analysis and Design of Power Electronic Transformer for Medium Voltage Levels," IEEE Power Electronics Specialists Conference, 2006, pp. 1-5.

[3] H. Iman-Eini, Sh. Farhangi, J-L Schanen and J. Aime, "Design of Power Electronic Transformer based on Cascaded H-bridge Multilevel Converter," IEEE International Symposium on Industrial Electronics, ISIE 2007, pp. 877-882, June 2007.

[4] H. Mirmousa and M. R. Zolghadri, "A Novel Circuit Topology for Three-Phase Four-Wire Distribution Electronic Power Transformer," International Conference on Power Electronics and Drive Systems, 2007 pp.1215-1222.

[5] R. Petkov, "Optimum Design of a High-Power, High-Frequency Transformer," IEEE Trans. Power Electronics, Vol. 11, No. 1, pp. 3342, Jan. 11996.

[6] William Gerard Hurley, Werner Hugo Wölfle and John G. Breslin, "Optimized Transformer Design: Inclusive of High-Frequency Effects," IEEE Trans. Power Electronics, Vol. 13, No. 4, pp.651-659, July 1998.

[7] Keith H. Billings, Switchmode Power Supply Handbook. McGraw-Hill, Inc. U.S.A., 1989, pp. 3.86-3.87.

[8] Colonel Wm. T. McLyman, Transformer and Inductor Design Handbook. Kg Magnetics, Inc. Idyllwild, California, U.S.A, 2004. 